\title{
Assessment of noninvasive positive pressure ventilation in healthy young volunteers using salivary stress biomarkers
}

Yuri Fueda*,1 (iD), Fuka Matsuda² \& Takuya Kataoka²

${ }^{1}$ Department of Medical Engineering, Faculty of Health Sciences, Morinomiya University of Medical Sciences, Osaka, Japan

${ }^{2}$ Department of Medical Engineering, Faculty of Health Sciences, Himeji Dokkyo University, Himeji, Hyogo, Japan

*Author for correspondence: Tel.: +81 661051 285; Fax: +81 647035 891; yuri_fueda@morinomiya-u.ac.jp

\begin{abstract}
Aim: We performed a stress assessment of noninvasive positive pressure ventilation (NPPV) using the salivary biomarkers chromogranin A. Materials \& methods: Twenty healthy volunteers were subjected to NPPV for 30 min. We collected saliva samples before and after NPPV and evaluated chromogranin A. Results: We collected saliva samples from 13 volunteers for enzyme measurement. Of the 13 volunteers, 11 showed elevated chromogranin A levels, which were significantly higher after NPPV than before NPPV ( $p$ $<0.01$ ). The chromogranin A increase group displayed significantly increased leak flow and reduced respiratory rate and absolute humidity compared with the chromogranin A reduction group. Conclusion: The increase of leak volume might be a stress factor in patients receiving NPPV.
\end{abstract}

Lay abstract: Salivary biomarkers are attractive as they are noninvasive and convenient. Noninvasive positive pressure ventilation (NPPV), the delivery of mechanical ventilation to those with respiratory failure, can have complications resulting in stress to the patient. Here, we evaluated stress-related salivary biomarkers during NPPV in healthy individuals. Our results suggest that NPPV might increase stress levels and that humidity and leak volume affected stress.

First draft submitted: 26 November 2019; Accepted for publication: 10 January 2020; Published online: 24 February 2020

Keywords: amylase $\bullet$ chromogranin A $\bullet$ humidity $\bullet$ leak volume $\bullet$ mask $\bullet$ noninvasive ventilation $\bullet$ saliva

The number of studies assessing stress using salivary biomarkers has been increasing because biomarker assessment is noninvasive, occasional and convenient [1]. Stress increases the activity of the sympathetic-adrenal nervous system, which is involved in the release of catecholamines from the adrenal medulla and in the activation of the hypothalamus-pituitary-adrenal axis [2]. The activation of these two responses is associated with changes in salivary biomarkers, such as cortisol, amylase and chromogranin A. For example, salivary amylase levels have been reported to significantly increase due to the calculation load [3]. Hill et al. reported that exercise increases plasma cortisol levels [4]. In addition, Filaire $e t$ al. demonstrated that public speaking reportedly increased salivary amylase activity and cortisol levels, but it did not cause any changes in chromogranin A levels [5]. Cortisol is an endocrine hormone that is secreted from the adrenal cortex via the hypothalamus-pituitary-adrenal axis in response to physical stress [1]. Amylase is a salivary enzyme and chromogranin A is an acidic glucoprotein. These two biological markers, which can also serve as psychological markers, are produced by catecholamines released from the adrenal medulla before the activation of the sympathetic nervous system [6-8]. Because they are also distributed in the submandibular gland, they are secreted into the saliva by sympathetic nervous stimuli (Figure 1).

Noninvasive positive pressure ventilation (NPPV) has an open-circuit design. The delivered flow of gas increases to compensate for the leak volume in the system [9-11]. However, a high flow causes complications, such as ear and sinus pain, gastric insufflation, ulcers, upper airway dryness and eye irritation [12,13]. The present study performed a stress assessment of NPPV using the salivary biomarkers chromogranin A and amylase. 


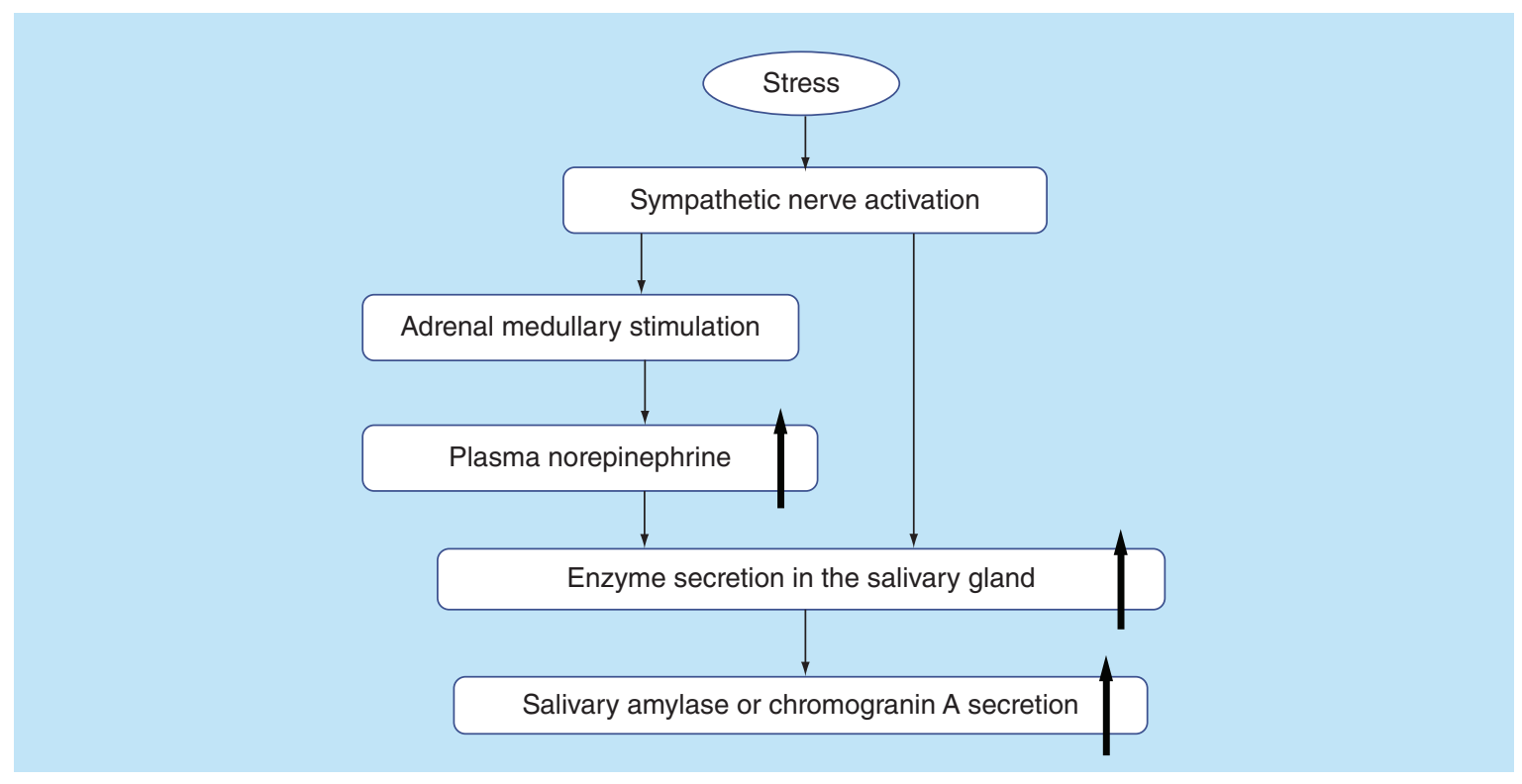

Figure 1. Mechanism of salivary enzyme secretion under stress. Levels of chromogranin A and amylase, as psychological stress biomarkers, increase in two pathways, which are involved in the activity of the sympathetic-adrenal nervous system as well as the activation of the salivary gland via direct stimulation of the sympathetic nervous [1,2].

\section{Table 1. Volunteer breathing function test results.}

\begin{tabular}{ll}
\hline Male/female, no. & $15 / 5$ \\
\hline Mean age (year) & $20.95 \pm 0.83$ \\
\hline Height $(\mathrm{cm})$ & $165.35 \pm 9.56$ \\
\hline Weight $(\mathrm{kg})$ & $61.87 \pm 19.79$ \\
\hline FVC $(\%)$ & $103.45 \pm 14.94$ \\
\hline FEV $1.0 \%$ & $103.04 \pm 9.52$ \\
\hline $\begin{array}{l}\text { Values are the median } \pm \text { standard deviation. } \\
\text { FEV: Forced expiratory volume; FVC: Forced vital capacity. }\end{array}$ \\
\hline
\end{tabular}

\section{Materials \& methods \\ Study setting}

This study was conducted at Himeji Dokkyo University from 2015 to 2016. The study protocol was reviewed and approved by the Himeji Dokkyo University Ethics Committee (number: 15-03) and all participants provided verbal consent.

\section{Volunteers}

We conducted breathing function tests on 20 college students (males: 15; females: 5 [Table 1]). These volunteers were healthy with no systemic illness and no ongoing medical treatment. They had forced vital capacity of $80 \%$ or more and forced expiratory volume of $70 \%$ or more in breathing function test. We excluded students with breathing function tests outside of the normal range and medical history. We verbally explained the purpose of the experiment to obtain consent before starting this study.

\section{Equipment}

NPPV was provided using a bi-level positive airway pressure ventilator (BiPAP vision, Philips Respironics, PA, USA). The ventilator circuits comprised a standard $180-\mathrm{cm}$ length of smooth-bore tubing, a heated humidifier (PMH1000, Pacific Medico, Tokyo, Japan) and an exhalation port. The interface comprised an optimal oronasal mask (Teijin, Tokyo, Japan) pressed against the volunteer's face. The ventilator settings were as follows: spontaneous and timed mode for $30 \mathrm{~min}$, with an inspiratory positive airway pressure of $10 \mathrm{~cm} \mathrm{H}_{2} \mathrm{O}$, an expiratory positive 
Figure 2. Chromogranin A before and after noninvasive positive pressure ventilation. The average levels of chromogranin A before and after NPPV were $0.612 \pm 0.314$ and $0.793 \pm 0.304 \mathrm{pmol} / \mathrm{mL}$, respectively. Chromogranin A levels were significantly influenced by NPPV.

NPPV: Noninvasive positive pressure ventilation.

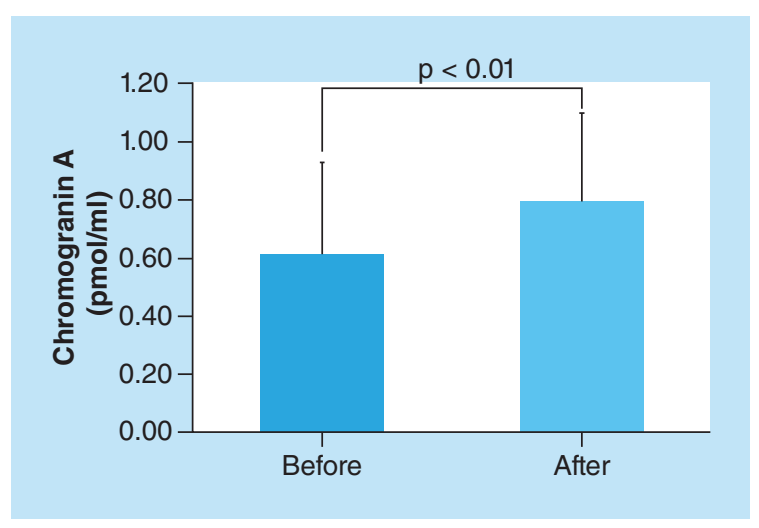

airway pressure of $5 \mathrm{~cm} \mathrm{H}_{2} \mathrm{O}$, a respiratory rate of 12 breaths/min and an inspired oxygen $\left(\mathrm{FiO}_{2}\right)$ rate of $21 \%$. We confirmed the exhaled ventilation volume, leak volume and respiratory rate on the ventilator monitor.

The following two different temperatures of a heated humidifier were assessed: medium and maximum degrees. The absolute humidity of the inspired gas during NPPV was measured using a temperature and humidity monitor (MAPHY+, Skynet, Osaka, Japan).

A pulse oximeter was attached to the volunteer's figure to measure heart rate and blood oxygen levels (SpO2). A multi-monitoring system (DS-7300, Fukuda Denshi, Tokyo, Japan) was used to measure heart rate and $\mathrm{SpO}_{2}$.

\section{Sample collection \& salivary biomarker analysis}

We used Salivette ${ }^{T M}$ cotton swabs (Sarstedt, Tokyo, Japan) to collect saliva before and after NPPV. The Salivette cotton swab was placed in the right or left cheek for $90 \mathrm{~s}$ to absorb saliva before NPPV. In the case of the end of NPPV, cotton swab was immediately placed in the same cheek as before NPPV for $90 \mathrm{~s}$. We instructed volunteers to let saliva absorb with cotton swab. The samples were centrifuged for $2 \mathrm{~min}$ at $3000 \mathrm{~g}$ at $4^{\circ} \mathrm{C}$ and the protein content was quantified with the BCA Protein Assay Kit (Takara Bio Inc., Shiga, Japan) after collection. The protein was standardized to a concentration of $50 \mu \mathrm{g} / \mathrm{ml}$ and the samples were stored at $-80^{\circ} \mathrm{C}$ to minimize protein activity until the salivary biomarkers were measured.

Salivary amylase activity was measured using a portable salivary amylase monitor (Nipro Co., Osaka, Japan) [14]. In general, a test strip was placed directly under the volunteer's tongue for approximately $30 \mathrm{~s}$ to absorb saliva and was then entered into the monitor. Salivary amylase finally appeared after $60 \mathrm{~s}$ [4]. However, the salivary amylase was not measured because it was not absorbed in the saliva in the cotton swab. To overcome this complication, we measured precisely $28 \mu \mathrm{l}$ of saliva according to the method by Yamaguchi et al. and dropped the samples on the tip [3].

Salivary chromogranin A levels were determined using enzyme immunoassay kits (YK070 Human Chromogranin A EIA kit, Yanaihara Institute, Inc., Shizuoka, Japan).

\section{Statistical analysis}

The results were described as the means \pm standard deviation. The differences were analyzed using paired $t$-tests and Wilcoxon signed rank test. Comparisons between groups were analyzed using Student's t-test. All statistical differences were considered significant with a p-value of $<0.05$. All statistical analyses were performed using Statcel 2 (OMS Ltd, Saitama, Japan).

\section{Results}

\section{Assessment of salivary biomarkers}

We were able to collect a sufficient amount of saliva from 13 of the 20 volunteers to assess salivary biomarkers. To compare before and after NPPV, we used samples from volunteers collected before and after NPPV and others were excluded.

Chromogranin A levels were elevated in 11 of the 13 volunteers. The average levels of chromogranin A before and after NPPV were 0.612 and $0.793 \mathrm{pmol} / \mathrm{ml}$, respectively. Chromogranin A levels were significantly influenced by NPPV ( $p<0.01$, Figure 2 ), whereas amylase activity was not responsive to NPPV. The average amylase activities before and after NPPV were 21.07 and $19.13 \mathrm{KU} /$, respectively (Figure 3). Amylase activities increased in five 

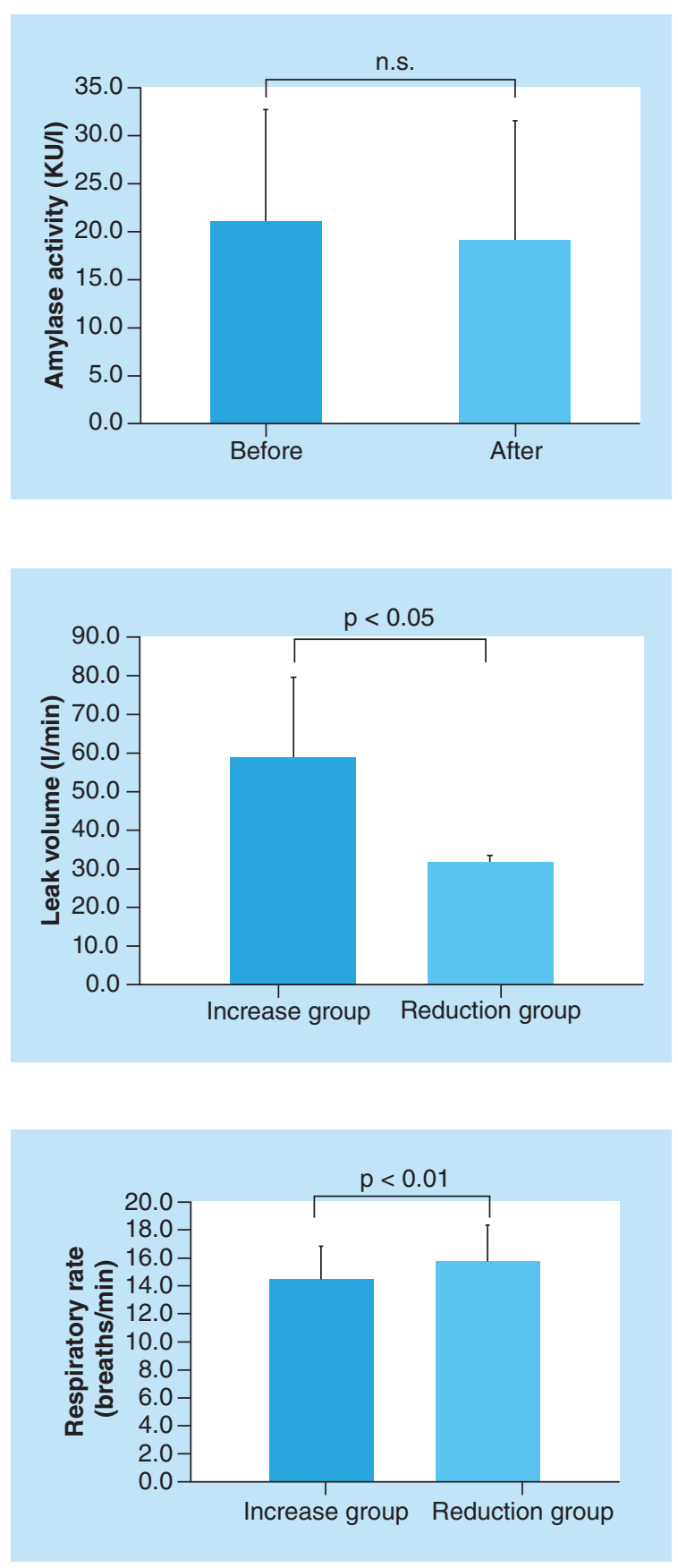

Figure 3. Amylase activity before and after noninvasive positive pressure ventilation. The average amylase activities before and after NPPV were $21.07 \pm 11.7$ and $19.13 \pm 12.4 \mathrm{KU} / \mathrm{l}$, respectively. There was no difference in amylase activity between before and after NPPV (n.s.). NPPV: Noninvasive positive pressure ventilation; n.s: Not significant.

Figure 4. Association between chromogranin A and leak volume. Leak volumes in the chromogranin $A$ increase group and the chromogranin $A$ reduction group were $58.64 \pm 41.37$ and $31.5 \pm 3.38 \mathrm{l} / \mathrm{min}$, respectively. The difference in leak volumes between the increase and reduction groups was statistically significant $(p<0.05)$.

Figure 5. Association between chromogranin A and respiratory rate. Respiratory rates in the chromogranin $A$ increase group and the chromogranin A reduction group were $14.72 \pm 2.25$ and $17.95 \pm 2.65$ breaths/min, respectively. The difference in respiratory rates between the increase and reduction groups was statistically significant $(p<0.05)$.

volunteers and decreased in seven volunteers. One volunteer did not display any changes in amylase activity.

\section{Association between chromogranin A \& NPPV parameters}

The leak volumes in the chromogranin $\mathrm{A}$ increase group and the chromogranin A reduction group were $58.64 \pm 41.37$ and $31.5 \pm 3.38 \mathrm{l} / \mathrm{min}$, respectively. The respiratory rates in the chromogranin $\mathrm{A}$ increase group and the chromogranin A reduction group were $14.72 \pm 2.25$ and $17.95 \pm 2.65$ breaths/min, respectively. The differences between the increase and reduction groups were statistically significant $(\mathrm{p}<0.05$; Figures $4 \& 5)$. In addition, the absolute humidity was higher in the reduction group than in the increase group. However, the difference in absolute humidity between the increase and reduction groups could not be statistically analyzed because there were not enough samples in the reduction group (Figure 6). The other parameters are described in Table 2. No other parameters affected chromogranin A levels. 
Figure 6. Association between chromogranin A and absolute humidity. Absolute humidity was higher in the reduction group $(24.53 \mathrm{mg} / \mathrm{l})$ than in the increase group $(18.08 \pm 4.63 \mathrm{mg} / \mathrm{l})$. However, the difference in absolute humidity between the increase and reduction groups could not be analyzed statistically since there were not enough samples in the reduction group.

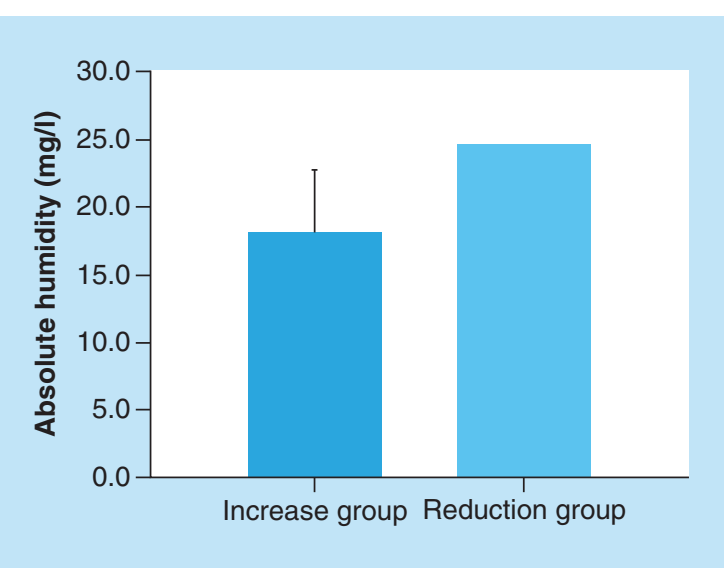

Table 2. Relationship between chromogranin A and parameters in noninvasive positive pressure ventilation $(\mathrm{n}=13)$. Items Increase group Reduction group p-value

Increase group exhaled volume $(\mathrm{ml}) \quad 781.71 \pm 466.94 \quad 598.35 \pm 209.56 \quad$ n.s.

$\begin{array}{llll}\mathrm{SpO}_{2}(\%) & 98.78 \pm 0.98 & 99.75 \pm 0.44 & \text { n.s. }\end{array}$

Heart rate $($ rate $/ \mathrm{min}) \quad 79.85 \pm 11.46 \quad 74.05 \pm 17.4 \quad$ n.s.

Values are the median \pm standard deviation

n.s.: Not significant.

Figure 7. Association between chromogranin A and inspired gas humidity. Absolute humidity at the medium

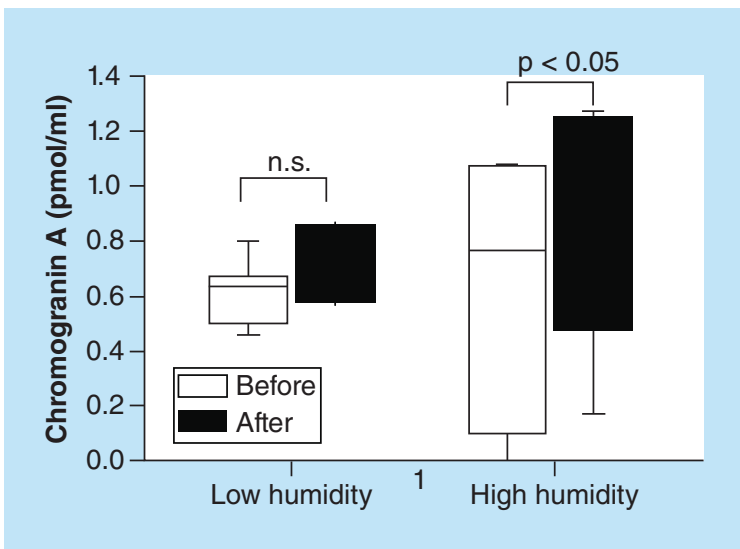
and maximum temperatures was $14.64 \pm 3.02$ and $22.21 \pm 1.75 \mathrm{mg} / \mathrm{ml}$, respectively. Chromogranin A levels were not responsive to low inspired gas humidity, whereas chromogranin A levels were significantly influenced by high inspired gas humidity. n.s.: Not significant.

\section{Association between chromogranin A \& inspired gas humidity}

The absolute humidity at the medium and maximum temperatures was $14.6 \pm 3.02$ and $22.20 \pm 1.75 \mathrm{mg} / \mathrm{ml}$, respectively. Chromogranin A levels were not responsive to low inspired gas humidity, whereas chromogranin A levels were significantly influenced by high inspired gas humidity $(\mathrm{p}<0.05$; Figure 7 ). 


\section{Discussion}

NPPV has been shown to be effective for patients with respiratory failure, heart failure and sleep apnea syndrome [1517]. NPPV can be immediately applied to patients to supply gas via nasal or full-face masks without undergoing tracheal intubation. However, the patients can easily remove the masks, so they may do so and not receive assistance with respiration if NPPV were a stress factor. NPPV provides pressure-controlled ventilation by changing the flow volume to compensate for leaks between the face and mask. A large gap between the face and mask may result in redness and ulcer development. If the flow volume increases due to increasing leak volume, the patient may experience discomfort. Therefore, cooperation between the patients and medical staff is essential. Before starting NPPV, we must explain its risks and benefits to patients and families.

In this study, we used the salivary biomarkers chromogranin A and amylase to assess stress. Chromogranin A is widely distributed in endocrine cells and the nervous system, particularly in the adrenal medulla and pituitary $[1,2]$. Chromogranin A is also produced by the submandibular gland and has been shown to be secreted into saliva by autonomic nerve stimulation [6]. Chromogranin A has a higher sensitivity to psychological stress than to exercise stress [18]. Nakane et al. reported that chromogranin A levels were elevated immediately before oral presentations and decreased immediately after the presentation for 15 min, whereas salivary chromogranin A levels may not be significantly influenced by $50 \mathrm{~min}$ of driving. We confirmed that the saliva collection time was optimal in this study. In another study, elevated norepinephrine levels were associated with elevated salivary amylase levels [19]. Norepinephrine activity is controlled by the sympathetic adrenal medullary system. In addition, amylase reacts promptly because it is directly acted upon by the sympathetic nervous system [1]. We assumed that our results were able to assess stress levels from saliva collected 2 min after NPPV completion.

The average chromogranin A levels were significantly different before and after NPPV; however, amylase activity did not change. Similar to our results, other reports have shown that the chromogranin A changes were inconsistent with those of amylase. Guilhem et al. reported that the chromogranin A levels and amylase activity showed different changes in swimmers than in other competitive sports [20]. Shinjo et al. reported that chromogranin A level decreased and amylase activity increased in the calculation load [21]. They showed that it was necessary to assess individual changes. Murakami et al. associated amylase activity with feelings such as comfort or anxiety. High-level volunteers according to the State-Trail Anxiety Inventory, which was used as an index of the state of anxiety, showed higher amylase activity than low-level volunteers [22]. Therefore, chromogranin A was used to evaluate psychological stress in this study. We assumed that amylase activity can be used to distinguish between the psychological stress levels of comfort and anxiety.

To the best of our knowledge, there have been no previous reports assessing stress in NPPV. Takashima et al. investigated the stressful experiences of ICU patients who were on artificial respirators for $12 \mathrm{~h}$ or longer [23]. Samuelson et al. reported ICU patients who underwent mechanical ventilation for more than $24 \mathrm{~h}$ had unpleasant and pleasant memories [24]. These authors suggested that the stressful experience of the ICU stay was associated with tracheal intubation. Tracheal intubation induces psychological stress, which is triggered by conversation difficulties and the physical restraint of movement. NPPV can assist patient breath without undergoing tracheal intubation. Therefore, we presumed that the stress factors in NPPV and mechanical ventilation will vary. The chromogranin A increase group displayed a significantly increased leak flow and reduced absolute humidity compared with the chromogranin A reduction group. Oto et al. associated leak flow with absolute humidity [25]. Leak flow increases the gas flow delivered by NPPV, which supplies an increased proportion of dry fresh gas for mixing with expired gas. Another report also showed that relative humidity decreased with increasing gas flow delivered by NPPV [26]. Those authors suggested that a high flow volume released warm, humid air through an exhalation port. We postulated that the chromogranin A reduction group experienced tension and anxiety before NPPV. The group with decreased chromogranin A levels had become accustomed to receiving NPPV for $30 \mathrm{~min}$.

Presently, it is not essential to use a heated humidifier in the NPPV system, according to respiratory guidelines [27]. NPPV delivers inspired gas through the upper airway, which provides $75 \%$ of the heat and moisture supplied to the alveoli. However, a heated humidifier is often used in NPPV to prevent problems such as dryness and throat congestion. We evaluated psychological stress with low and high levels of absolute humidity. The two absolute humidity levels evaluated were not significantly different. Therefore, we presumed that an absolute humidity of more than $15 \mathrm{mg} / \mathrm{l}$ is necessary. Wiest et al. reported that an absolute humidity of less than $10 \mathrm{mg} / \mathrm{l}$ was associated with upper airway dryness in continuous positive airway pressure (CPAP) users [28]. By contrast, 50\% of NPPV patients experienced severe oral dryness regardless of the absolute humidity level when it was more than $30 \mathrm{mg} / \mathrm{lon}$ 
average [25]. The patients suffered from acute respiratory failure and elicited more oral dryness, resulting in higher respiratory rates, increased mouth breathing and excessive immune responses compared with healthy volunteers. On the basis of our study, an absolute humidity level more than $15 \mathrm{mg} / \mathrm{l}$ is desirable. Nevertheless, we may need to adjust absolute humidity if the patient's condition is poor.

We evaluated psychological stress by focusing on pressure and delivered flow volume in NPPV. Previous reports have shown that redness and ulcers are caused, in part, by the strong contact between the face and mask such as on the forehead, nasal bridge and cheeks $[12,13]$. The incidence of pressure ulcers was higher with a nasal mask than with a full-face mask.

We suppose that the mask itself is also a stress factor. There are several types of masks, such as nasal-oral, full-face and helmet [29]. It is important, but challenging, for medical workers to control masks according to the patient's face size. Therefore, the psychological stress associated with many masks must be examined.

\section{Conclusion}

NPPV led to the elevation of psychological stress biomarker levels in healthy individuals. In addition, salivary chromogranin A levels were a more sensitive stress biomarker than amylase levels. The tested stress biomarkers were affected by change in absolute humidity. These results indicate that decreased absolute humidity increases the inspiratory flow depending on the leak volume. We propose that leak volume can serve as a psychological stress factor during NPPV in healthy individuals.

\section{Future perspective}

In this study, volunteers were young and healthy. However, we need to understand the stress status of patients undergoing respiratory induction. At present, we have constructed a pathological model and evaluated stress. Stress assessment surveys have been conducted in additional experiments on stress evaluation using questionnaire and fingertip pulse waves. If we can construct an experimental method, we will seek further patients and collaboration with other institutions in our proceeding study.

\section{Summary points}

- Saliva biomarkers were used for assessment of stress caused by noninvasive positive pressure ventilation.

- Saliva samples could be noninvasively collected.

- To investigate the cause of the stress, leak flow, the inspiratory flow and absolute humidity were measured.

- Of the 13 volunteers, 11 showed elevated chromogranin A levels.

- Chromogranin A is more sensitive than amylase.

- The chromogranin A increase group displayed significantly increased leak flow and reduced respiratory rate and absolute humidity compared with the chromogranin A reduction group.

- It was important to control leak flow during noninvasive positive pressure ventilation.

- Stress caused by the use of other interfaces and pathological models would also need to be assessed.

\section{Authors' contributions}

Y Fueda was responsible for literature search, data collection, study design, data analysis and manuscript preparation; F Matsuda was responsible for study design, data collection and data analysis; T Kataoka was responsible for study design, data collection and data analysis.

\section{Acknowledgments}

We are thankful to the individuals from Himeji Dokkyo University who participated in this study as well as the clinical engineers at Velitas Hospital for their advice and for allowing us to use their equipment. We would also like to thank Enago (www.enago.jp) for the English language review.

\section{Financial \& competing interests disclosure}

The work was supported by special research grants from Himeji Dokkyo University (\#705 and \#737). The authors have no other relevant affiliations or financial involvement with any organization or entity with a financial interest in or financial conflict with the subject matter or materials discussed in the manuscript apart from those disclosed.

No writing assistance was utilized in the production of this manuscript. 


\section{Ethical conduct of research}

The authors state that they have obtained appropriate institutional review board approval or have followed the principles outlined in the Declaration of Helsinki for all human or animal experimental investigations. In addition, for investigations involving human subjects, informed consent has been obtained from the participants involved.

\section{Open access}

This work is licensed under the Creative Commons Attribution 4.0 License. To view a copy of this license, visit http://creativecomm ons.org/licenses/by/4.0/

\section{References}

Papers of special note have been highlighted as: $\bullet$ of interest; $\bullet \bullet$ of considerable interest

1. Papacosta E, Nassis GP. Saliva as a tool for monitoring steroid, peptide and immune markers in sport and exercise science. J. Sci. Med. Sport. 14(5), 424-434 (2011).

-• Review on stress assessment using the salivary biomarkers.

2. Chrousos GP, Gold PW. The concepts of stress and stress system disorders. Overview of physical and behavioral homeostasis. JAMA 267(9), 1244-1252 (1992).

-. Overview of hormones for the stress assessment.

3. Yamaguchi M, Kanemori T, Kanemaru M, Mizuno Y, Yoshida H. Correlation of stress and salivary amylase activity. JJME 39(3), 234-239 (2001).

-• Simple method for measuring amylase levels.

4. Hill EE, Zack E, Battaglini C, Viru M, Viru A, Hackney AC. Exercise and circulating cortisol levels: the intensity threshold effect. J. Endocrinol. Invest. 31(7), 587-591 (2008).

5. Filaire E, Dreux B, Massart A, Nourrit B, Rama LM, Teixeira A. Salivary alpha-amylase, cortisol and chromogranin A responses to a lecture: impact of sex. Eur. J. Appl. Physiol. 106(1), 71-77 (2009).

6. Saruta J, Tsukinoki K, Sasaguri K et al. Expression and localization of chromogranin A gene and protein in human submandibular gland. Cells Tissues Organs 180(4), 237-244 (2005).

-• Specific location of chromogranin A.

7. Walsh NP, Blannin AK, Clark AM, Cook L, Robson PJ, Gleeson M. The effects of high-intensity intermittent exercise on saliva IgA, total protein and alpha-amylase. J. Sports Sci. 17(2), 129-134 (1999).

8. Crnković D, Peco M, Gelo J. Correlation between salivary biochemical stress indicators and psychological indicators. Acta Clin. Croat. 57(2), 316-326 (2018).

9. Mehta S, McCool FD, Hill NS. Leak compensation in positive pressure ventilators: a lung model study. Eur. Respir. J. 17, 259-267 (2001).

10. Ferreira JC, Chipman DW, Hill NS, Kacmarek RM. Bilevel vs ICU ventilators providing noninvasive ventilation: effect of system leaks: a COPD lung model comparison. Chest 136(2), 448-456 (2009).

11. Yamaguchi M, Hanawa N, Yoshida $\mathrm{H}$. Evaluation of a novel monitor for the sympathetic nervous system using salivary amylase activity. JSMBE 45(2), 161-168 (2007).

12. Gay PC. Complications of noninvasive ventilation in acute care. Respir. Care. 54(2), 246-257 (2009).

13. Schallom M, Cracchiolo L, Falker A et al. Pressure ulcer incidence in patients wearing nasal-oral versus full-face noninvasive ventilation masks. Am. J. Crit. Care 24(4), 349-356 (2015).

14. Chanques G, Constantin JM, Sauter M et al. Discomfort associated with underhumidified high-flow oxygen therapy in critically ill patients. Intensive Care Med. 35(6), 996-1003 (2009).

15. Kato T, Suda S, Kasai T. Positive airway pressure therapy for heart failure. World J. Cardiol. 6(11), 1175-1191 (2014).

16. Scala R, Pisani L. Noninvasive ventilation in acute respiratory failure: which recipe for success? Eur. Respir. Rev. 27(149), 180029 (2018).

17. Yamashita S, Dohi T, Narui K, Momomura S. Therapeutic efficacy of continuous positive airway pressure in obstructive sleep apnea patients with acute aortic dissection: a case report. J. Atheroscler. Thromb. 17(9), 999-1002 (2010).

18. Nakane H, Asami O, Yamada Y et al. Salivary chromogranin A as index of psychosomatic response. Biomed. Res. 19(6), 401-406 (1998).

- Sensitivity of the chromogranin A to psychological stress.

19. Lynch CD, Sundaram R, Maisog JM, Sweeney AM, Buck Louis GM. Preconception stress increases the risk of infertility: results from a couple-based prospective cohort study - the LIFE study. Hum. Reprod. 29(5), 1067-1075 (2014).

20. Guilhem G, Hanon C, Gendreau N, Bonneau D, Guével A, Chennaoui M. Salivary hormones response to preparation and pre-competitive training of world-class level athletes. Front. Physiol. 6(333), 1-9 (2015). 
21. Shinjo T, Sakuraba K, Nakaniida A et al. Oral lactoferrin influences psychological stress in humans: a single-dose administration crossover study. Biomed. Rep. 8(5), 426-432 (2018).

22. Murakami M, Tahara Y, Takeda K, Yamaguchi M. Psychosomatic stress and salivary amylase activity in junior high school student. JSMBE 47(2), 166-171 (2009).

23. Takashima N, Murata H, Nishikaichi Y, Yamaguchi Y, Sakai K, Takinami M. Stressful experiences in the ICU of patients put on mechanical ventilators for 12 hours or more. J. Jpn Soc. Intensive Care Med. 24(4), 399-405 (2017).

24. Samuelson KA. Unpleasant and pleasant memories of intensive care in adult mechanically ventilated patients-findings from 250 interviews. Intensive Crit. Care Nurs. 27(2), 76-84 (2011).

25. Oto J, Nakatani E, Okuda N, Onodera M, Imanaka H, Nishimura M. Hygrometric properties of inspired gas and oral dryness in patients with acute respiratory failure during noninvasive ventilation. Respir. Care 59(1), 39-45 (2014).

- Relationship between leak and humidity.

26. Holland AE, Denehy L, Buchan CA, Wilson JW. Efficacy of a heated passover humidifier during noninvasive ventilation: a bench study. Respir. Care 52(1), 38-44 (2007).

-• Importance of humidifier during noninvasive positive pressure ventilation.

27. , Restrepo RD, Walsh BK. Humidification during invasive and noninvasive mechanical ventilation: 2012. Respir. Care 57(5), 782-788 (2012).

28. Wiest GH, Foerst J, Fuchs FS, Schmelzer AH, Hahn EG, Ficker JH. In vivo efficacy of two heated humidifiers used during CPAP-therapy for obstructive sleep apnea under various environmental conditions. Sleep 24(4), 435-440 (2001).

29. Nava S, Navalesi P, Gregoretti C. Interfaces and humidification for noninvasive mechanical ventilation. Respir. Care 54(1), 71-84 (2009). 